\title{
Our professional opinion
}

\author{
Professional editors provide the perspective, consistency and responsiveness needed to identify and \\ communicate groundbreaking scientific advances.
}

Scientific publishing developed as a way to communicate scientific discoveries to peers. By first simply collecting and later evaluating contributions, scientists were able to avoid duplicating the efforts of others and instead build directly on prior results. In the modern era, all journals make use of this same basic framework, but the ways in which papers are evaluated and the models of how papers are published vary widely. As each system has advantages and disadvantages, new models continue to arise to address real or perceived limitations of existing approaches. Within this context, we submit that journals coordinated by professional editors offer unique and important advantages to the scientific community.

A professional editor acts as an in-house expert in and advocate for specific fields or subfields within the overall scientific scope of their journal. To improve their knowledge of ongoing efforts and challenges in their fields, and to promote communication within and across fields, editors attend conferences in their topic areas, engage with members of the community and commission reviews or other highlights of important work. Editors also take primary responsibility for submitted manuscripts in their fields, communicating with authors at all steps from an initial editorial decision through subsequent decisions on reviewed manuscripts and final acceptance through to publication of the paper.

The initial assessment of a manuscript requires a careful evaluation of its merits in regards to the field as a whole, and occupies a significant proportion of an editor's time. Whereas academic editors may consider a paper based on more first-hand knowledge of a specific subfield or experimental technique, professional editors are able to draw from a different set of information in making their decisions. For example, though professional editors are $\mathrm{PhD}$-level scientists (usually with postdoctoral research experience or beyond) and thus have an appropriate scientific framework to understand and think critically about a manuscript's contents, their position outside of a particular subfield affords a broader scientific perspective from which to evaluate the importance of any one result. This external but engaged view also permits a level of editorial independence that decouples an author's track record or influence from the decision process. Additionally, because professional editors see a wide range of referee feedback and actively seek community guidance for establishing standards in a given field, they are well placed to recognize the type of discoveries likely to find support from external experts and the lines of evidence needed to support particular claims. By pursuing only those manuscripts that potentially offer unprecedented insights and contain appropriate experimental support, editors can provide timely feedback to the large majority of authors whose manuscripts are returned to them without review and focus their attention on manuscripts likely to be of greatest interest to the journal's readership.

Although we are sympathetic to authors' frustrations that only a minority of papers are sent for peer review, we are perplexed by the occasional criticism that professional editors are unsuited to evaluate manuscripts. Indeed, 'triaging' papers is something that scientists do on a daily basis in selecting which articles in the literature they will read in full, in part or not at all. With the increasing volume of publications, even the use of Internet search engines and associated keyword-based alerts highlight more potentially relevant papers than can realistically be read by a single scientist. Professional editors thus effectively prescreen papers by acting not only as scientists but also as advocates of the scientific readership, considering papers in several dimensions in an effort to ensure that all papers published in the journal will be required reading for the field.

How do we 'screen' papers? Contrary to suggestions that professional editors are indecisive and act merely as managers (Nature 472, 391, 2011), editors can only be effective if they form thoughtful opinions and act accordingly. Beyond the initial evaluation, in which editors must be able to enumerate the strengths of a particular manuscript, editors take an active role in managing the peer review process. As with academic editors, professional editors rely on referees to evaluate the technical merits of a manuscript, weigh in on whether the experimental evidence supports the major conclusions of a study and provide advice as to whether the findings are sufficient to merit publication in the journal (Nat. Chem. Biol. $6,245,2010)$. However, a paper for which all three referees provide consistent advice that points to a clear decision is the exception, not the rule, particularly in interdisciplinary fields. As such, professional editors do not simply hand referee reports back to authors: editors consider referee feedback, provide guidance for revisions and moderate technical disagreements that arise (Nat. Chem. Biol. 4, 715, 2008; Nat. Chem. Biol. $7,1,2011)$. In addition, much like a grant review panel that can only fund a subset of high-quality proposals, editors weigh referee enthusiasm for any one manuscript against the comments for others to strategically select representative papers from across the fields they cover and publish only the most compelling manuscripts.

We agree that no publication model is perfect. However, we submit that professional editors' pursuit of a single objective-to find and publish the best scientific papersprovides a strong correlation with the success of a particular journal according to almost any measure. This is in part a result of the additional support provided to authors that adds value to and raises the profile of their manuscripts, including artistic support, copyediting, technical proofreading and press coverage. Streamlining the number of editors making decisions to a few fulltime professionals who work as a team also promotes consistency and fairness as well as the ability to alter editorial criteria as standards evolve or paradigm-changing discoveries are made. We further posit that wider adoption of this model would decrease the prevalence of 'least publishable units', which are causing strain on authors, referees and funding agencies (Nature 463, 1009, 2010; Nat. Chem. Biol. 6, 307, 2010).

At Nature Chemical Biology, we continually challenge ourselves to keep connected with current research and be mindful of the evolution of the fields we cover. We strive to make our decisions timely and transparent for authors so that, even if they are unsuccessful with one submission, the insight they gain into our processes could benefit future submissions. Finally, we are aware that publishing models continually evolve (Nat. Chem. Biol. 2, 391, 2006), and we seek opportunities for improving the publishing process through the open dialogue we consistently pursue with our authors, referees and readers. 